\title{
Development of a Multifidelity Approach to Acoustic Liner Impedance Eduction
}

\author{
Douglas M. Nark* and Michael G. Jones ${ }^{\dagger}$ \\ NASA Langley Research Center, Hampton, VA 23681-2199, U.S.A
}

\begin{abstract}
The use of acoustic liners has proven to be extremely effective in reducing aircraft engine fan noise transmission/radiation. However, the introduction of advanced fan designs and shorter engine nacelles has highlighted a need for novel acoustic liner designs that provide increased fan noise reduction over a broader frequency range. To achieve aggressive noise reduction goals, advanced broadband liner designs, such as zone liners and variable impedance liners, will likely depart from conventional uniform impedance configurations. Therefore, educing the impedance of these axial- and/or spanwise-variable impedance liners will require models that account for three-dimensional effects, thereby increasing computational expense. Thus, it would seem advantageous to investigate the use of multifidelity modeling approaches to impedance eduction for these advanced designs.

This paper describes an extension of the use of the CDUCT-LaRC code to acoustic liner impedance eduction. The proposed approach is applied to a hardwall insert and conventional liner using simulated data. Educed values compare well with those educed using two extensively tested and validated approaches. The results are very promising and provide justification to further pursue the complementary use of CDUCT-LaRC with the currently used finite element codes to increase the efficiency of the eduction process for configurations involving three-dimensional effects.
\end{abstract}

\section{Introduction}

Increasing air traffic and more stringent aircraft noise regulations continue to expand noise reduction requirements for conventional and unconventional aircraft configurations. One of the dominant component noise sources for subsonic aircraft is the fan noise produced by the engine. Therefore, significant effort has been directed toward reducing this noise component both at its source and along transmission/radiation paths. As an approach to reducing fan noise transmission/radiation, the use of acoustic liners has proven to be extremely effective. However, the introduction of advanced fan designs and shorter engine nacelles has highlighted a need for novel acoustic liner designs that provide increased fan noise reduction over a broader frequency range.

The key parameter used to understand noise reduction achieved with an acoustic liner is acoustic impedance, an intrinsic parameter that is dependent on sound pressure level and grazing flow velocity. As discussed by Jones and Watson, 1 a number of methods have been developed to determine acoustic impedance in the presence of grazing flow. These range from direct measurement approaches (e.g., in situ microphones/probes, Laser Doppler Velocimetry) to impedance eduction methods based on a combination of duct propagation code and measurements within the hardwall sections (upstream/downstream or opposite) of a liner mounted in the flow duct. The NASA Langley Liner Physics Team has investigated a number of these impedance eduction methods with a particular focus on the latter approaches. These have been based on three sets of equations; the convected Helmholtz equation, limited to uniform background flow, and the linearized Euler equations and the Pridmore-Brown equation, which include shear-flow effects $\sqrt[23]{ }$ The methods based on the convected Helmholtz and linearized Euler equations assume a finite-length acoustic liner mounted in a rectangular waveguide. Here, the educed impedance value is iterated upon until the acoustic pressures predicted by a duct propagation code match the acoustic pressures measured at a number of microphone locations to within an acceptable tolerance. Alternatively, the method based on the Pridmore-Brown equation assumes an infinite-length acoustic liner mounted in a rectangular waveguide. This approach, which is well suited to cases where a single mode is dominant over a significant portion of the liner, uses amplitude and phase decay rates over the axial extent of the liner to directly compute the liner impedance.

\footnotetext{
* Senior Research Scientist, Structural Acoustics Branch, Research Directorate, AIAA Associate Fellow

${ }^{\dagger}$ Senior Research Scientist, Structural Acoustics Branch, Research Directorate, AIAA Associate Fellow
} 
Previous studies based on these three impedance eduction methods have, in general, provided excellent results that continue to be used throughout the acoustic liner community. However, these applications have typically involved uniform impedance acoustic liners such that two-dimensional or quasi-three-dimensional models could be employed. To achieve the aforementioned noise reduction goals, advanced broadband liner designs will likely depart from conventional uniform impedance configurations (e.g., zone liners, variable impedance liners). Therefore, educing the impedance of these axial- and/or spanwise-variable impedance liners will require models that account for three-dimensional effects. While this capability exists with methods based on the convected Helmholtz and linearized Euler equations, an increase in computational requirements is inherently incurred.

One possible approach to alleviating this difficulty is to employ multiple propagation codes of varying levels of fidelity to reduce computational costs. For instance, the propagation module of the NASA Langley developed CDUCT-LaRC (CDL) code ${ }^{4}$ utilizes a parabolic approximation to the convected Helmholtz equation formulated by Dougherty ${ }_{516}^{5}$ This approach affords very efficient propagation calculations, thus allowing solutions for complex threedimensional geometries to be handled with relatively low computational costs. However, this efficiency can come at the expense of reduced fidelity as the actual propagation direction may diverge from the preferred direction of the parabolic approximation. Additionally, loss of fidelity may occur when reflection and/or scattering of acoustic waves become important. Nevertheless, if appropriate care is taken to account for the assumptions of the parabolic approximation, CDL provides an efficient framework in which to perform fully three-dimensional aeroacoustic computations. It has been successfully coupled with the optimization package of the SciPy ${ }^{7}$ library and used effectively in several broadband liner optimization studies ${ }^{8}-10$ Thus, it would seem appropriate to investigate the possible complementary use of CDL with the finite element codes employed in the eduction methods based on the convected Helmholtz and linearized Euler equations. For example, CDL could be used to efficiently identify approximate impedance values and the higher fidelity (albeit more computational intensive) methods could then be used to obtain more accurate results. Similarly, CDL and higher fidelity methods could be combined in a multifidelity modeling framework.

The purpose of the current work is to perform an initial investigation of the proposed use of CDL in impedance eduction. The test cases considered are selected to follow previous studies on impedance eduction methodologies $11-13$ and consist of a hardwall insert and a conventional perforate-over-honeycomb liner. The component results are used to validate the CDL impedance eduction approach for each of these liner configurations using simulated acoustic data in the NASA Grazing Flow Impedance Tube (GFIT) ${ }^{14 \mid 15}$ with and without grazing flow. A description of the duct geometry and test samples used in this investigation is provided in Section II Section III provides a description of the aeroacoustic propagation model. Results are provided in Section IV] and concluding remarks regarding some of the more significant results and further areas of interest are presented in Section $\mathrm{V}$

\section{Description of Test Configurations}

\section{A. Flow Duct}

Over the last three decades, NASA Langley Research Center has developed a number of test rigs for the evaluation of acoustic liners with and without grazing flow. For this initial study, the GFIT (see Fig. 1) was selected due to its extensive use in previous impedance eduction work $\frac{16}{19}-19$ and to limit the presence of higher modes in the analysis. The GFIT, where the surface of the test liner forms a portion of the upper wall of the flow duct, has a cross-sectional geometry of 2.0 " $(50.8 \mathrm{~mm})$ wide by 2.5 " $(63.5 \mathrm{~mm})$ high and allows evaluation of acoustic liners with lengths from 2.0" (50.8 $\mathrm{mm}$ ) to 24.0 " (609.6 $\mathrm{mm})$. The test liner is assumed to have an unknown, but uniform, normalized impedance, $\zeta$. Throughout this paper all impedances are normalized by the characteristic impedance, $\rho_{0} c_{0}$, of the air flowing in the duct (left to right in Fig. 11). Twelve acoustic drivers form an upstream (exhaust mode) source section. These drivers are used to generate tones (one frequency at a time) over a frequency range of 400 to $3000 \mathrm{~Hz}$, at source levels (measured at the reference microphone, located at $x=0.0$ ) up to $140 \mathrm{~dB}$, and at centerline Mach numbers ranging from 0.0 to 0.6 . Ninety-five (95) microphones are flush-mounted in the four walls of the GFIT, and are used to measure the acoustic pressure field over the spatial extent of $\{0 \leq x \leq L, 0 \leq y \leq H, 0 \leq z \leq W\}$, where $L=40$ " $(1016 \mathrm{~mm}), H=2.5$ " $(63.5 \mathrm{~mm})$, and $W=2.0$ " $(50.8 \mathrm{~mm})$ represent the length, height, and width, respectively, of the test section.

\section{B. Liner Configurations}

Two liner configurations, both of length 16 " $(406.4 \mathrm{~mm})$, are considered in the current investigation with properties as described below. 


\section{Hardwall Insert}

The first liner is a hardwall insert whose structure is composed of a $0.5 "(12.7 \mathrm{~mm})$ thick aluminum plate. The hardwall insert is chosen because it provides a liner for which the impedance is known a priori. It is noted that for this insert, the resistance and reactance values are very large. Thus, the results are presented in terms of the normalized admittance, $\beta=1 / \zeta$. Here, the admittance is decomposed into its real and imaginary parts, $\beta=\sigma+i \gamma$, where $\sigma$ and $\gamma$ are the normalized conductance and susceptance, respectively. The educed values of these parameters are expected to be zero for a perfectly rigid insert.

\section{Conventional Liner}

The second test liner is a conventional, single degree-of-freedom (SDOF), perforate-over-honeycomb liner as shown in Figure 2. The geometrical parameters associated with this liner (see Table 1) are representative of those commonly used in current aircraft engine nacelles. Additionally, this liner has been studied previously ${ }^{13}$ and educed impedance values from two extensively tested and validated approaches ${ }^{12 \mid 20}$ are available.

Table 1: Liner Geometric Parameters

\begin{tabular}{|c|c|c|c|}
\hline $\begin{array}{c}\text { Facesheet Thickness, } t \\
\text { in, }(\mathrm{mm})\end{array}$ & $\begin{array}{c}\text { Hole Diameter, } d \\
\text { in, }(\mathrm{mm})\end{array}$ & $\begin{array}{c}\text { Cavity Depth, } h \\
\text { in }(\mathrm{mm})\end{array}$ & Open Area Ratio, $\sigma$ \\
\hline $0.032(0.813)$ & $0.038(0.965)$ & $1.5(38.176)$ & 0.089 \\
\hline
\end{tabular}

\section{Impedance Eduction Methodology}

For the current investigation, duct propagation predictions are conducted using the CDUCT-LaRC (CDL) code. This code is typically used to predict the propagation of a given acoustic source ahead of the fan face or aft of the exhaust guide vanes in the inlet or exhaust ducts, respectively. The duct propagation module is based on the CDUCT code developed by Dougherty ${ }^{5 / 6}$ and extended by Lan ${ }^{[21}$ The CDL code has been extended ${ }^{4}$ to support multiblock propagation calculations, for which grid connectivity is determined and data transferred from upstream to downstream blocks without user intervention. The acoustic treatment is represented through an impedance boundary condition and may be allowed to vary in the axial and/or spanwise directions. As mentioned previously, CDL utilizes a parabolic approximation to the convected Helmholtz equation that affords very efficient propagation calculations, thus allowing solutions for complex three-dimensional geometries to be handled with relatively low computational costs. As such, it has been successfully coupled with the optimization package of the SciPy ${ }^{7}$ library and used effectively in several broadband liner optimization studies ${ }^{[8] 10}$ Therefore, with modification to the objective function, this optimization methodology can be applied to impedance eduction for configurations requiring models that account for three-dimensional effects. However, before proceeding to such cases, it is prudent to validate the approach with the more conventional configurations considered in this work.

Figure 3 provides a schematic of the three-dimensional flow duct and Cartesian coordinate system used in the computational domain. The surface of the liner with unknown normalized impedance, $\zeta$, comprises a portion of the upper wall of the flow duct over the axial extent of $L_{1} \leq x \leq L_{2}$. Upstream and downstream of the liner, the flow duct upper wall is rigid. The lower and two side walls are also rigid, and the source and exit planes of the computational domain are located at $x=0$ and $x=L$, respectively. Consistent with the GFIT measurements, the sound source is assumed to be a single tone plane wave with the amplitude and phase taken from the microphone located in the source plane ( $x=0.0$ in Figure 3). The acoustic pressure field is captured at fifty-three flush-mounted microphone locations in the lower wall (opposite the liner) over the axial extent of $0 \leq x \leq L$. The unknown normalized impedance, $\zeta$, is educed by an iterative process that determines the resistance, $\theta$, and reactance, $\chi$, that reproduces the acoustic wall pressures at the microphone locations. This is achieved by minimizing the objective function

$$
F(\theta, \chi)=\sum_{n=1}^{n w a l l}\left\|\left\{R_{\text {dif }}\right\}\right\|_{L 2},\left\{R_{\text {dif }}\right\}=\left\{\begin{array}{c}
p_{\text {mic }}\left(x_{1}\right)-p_{\text {pred }}\left(x_{1}\right) \\
p_{\text {mic }}\left(x_{2}\right)-p_{\text {pred }}\left(x_{2}\right) \\
\vdots \\
p_{\text {mic }}\left(x_{\text {nwall }}\right)-p_{\text {pred }}\left(x_{n \text { wall }}\right)
\end{array}\right\}
$$


where $n$ wall is the number of microphone locations (i.e., 53 for this investigation) and $\left\|\left\{R_{\text {dif }}\right\}\right\|_{L 2}$ denotes the L2norm of the complex residual error vector between the microphone data $\left(p_{\text {mic }}\right)$ and predicted value $\left(p_{\text {pred }}\right)$. A number of constrained optimization algorithms are available within the SciPy ${ }^{7}$ optimization package (e.g, gradient based, basin-hopping, differential evolution). In this case, the minimization is achieved using the limited-memory boundconstrained Broyden-Fletcher-Goldfarb-Shanno (L-BFGS-B) $22 \mid 23$ optimization algorithm, which results in the predicted normalized resistance, $\theta$, and normalized reactance, $\chi$, of the test liner. Among the input required by this optimization algorithm is the objective function convergence tolerance. This parameter controls the stopping criteria used to terminate the optimization and hence, the resultant educed impedance values.

\section{Results and Discussion}

The purpose of this initial study is to determine the general applicability of the CDL approach to impedance eduction, therefore, simulated data is used at the specified microphone locations. This provides greater control of input data and avoids shear layer effects and measurement uncertainties. Randomization is subsequently applied to the simulated data to replicate measurement uncertainty. The data is generated for the hardwall insert and conventional liner with a tonal source (one frequency at a time from $400-3000 \mathrm{~Hz}$ in $200 \mathrm{~Hz}$ increments) at an incident SPL of 120 $\mathrm{dB}$. For the hardwall insert, an admittance value of $\beta=0.0+0.0 i$ is used for all conditions. As mentioned in Section 2. educed impedance values from the $\mathrm{CHE}^{20}$ code and Kumaresan and Tufts algorithm (KT) ${ }^{12}$ are available. These approaches are described in detail in the cited references, so only those aspects that are key to the current study are provided herein. The CHE method of impedance eduction solves the convective Helmholtz equation using a finite element method. The impedance boundary conditions are incorporated in the FEM analysis in weak form and cubic Hermite polynomials are used as the basis functions. The unknown impedance of the test liner is then obtained by minimizing an objective function whose minimum occurs when the assumed test liner impedance is as close as possible to the true value. This is the general methodology followed in the CDL approach (described in Section [III), except for changes in the propagation code and optimization algorithms. In the KT approach, a single axial propagation constant is extracted from the measured, nonprogressive, multimodal, acoustic pressure field over the liner. This axial propagation constant is then used as input into the wall impedance boundary condition equation and normal mode solution to educe the liner impedance.

The CHE code is used to generate all simulated data with the admittance values for the hardwall insert as defined above and the impedance values for the conventional liner obtained from the previous CHE eduction process. The downstream termination is taken to be anechoic to avoid reflections. However, as the CHE code solves the convected Helmholtz equation, standing waves may still be present in the simulated data due to reflections from the leading and trailing edges of the liner. Alternatively, the CDL code employs a parabolic approximation to the convected Helmholtz equation and therefore, does not capture reflections due to impedance discontinuities at the leading and trailing edges of the liner. Examples of this behavior and the effect on the educed impedance are provided below.

For the gradient based optimization algorithm used in the CDL eduction methodology, initial values are required for the eduction quantities. For samples with very high impedance, such as the hardwall insert, the process is initialized using a normalized conductance of 0.8 (i.e., $\sigma=0.8$ ) and a normalized susceptance of 0.4 (i.e., $\gamma=0.4$ ). These values are also constrained throughout the optimization such that $10^{-8} \leq \sigma \leq 10.0$ and $-10.0 \leq \gamma \leq 10.0$. Alternatively, for samples with lower impedance values (i.e., conventional liners), the process is initialized using a normalized resistance of 1.0 (i.e., $\theta=1.0$ ) and a normalized reactance of -0.5 (i.e., $\chi=-0.5$ ). Further, the normalized resistance and reactance are constrained such that $0<\theta \leq 10.0$ and $-10.0 \leq \chi \leq 10.0$. These limits on $\theta$ and $\chi$ encompass values encountered for typical aircraft engine liners.

Based on the simulated data and constraints described above, the admittance of the hardwall insert and the impedance of the conventional liner are educed for each frequency and Mach number. The results are then compared with the corresponding result obtained via direct modeling and/or the use of CHE and KT. Educed admittances for the hardwall insert with no mean flow $(M=0.0)$ are shown in Figure 4 In this case, two CDL results are presented to illustrate the effects of the optimization convergence tolerance. As expected, if this parameter is too large, the educed admittance results do not match the expected hardwall values. When the convergence tolerance parameter is set to a sufficiently small value, the normalized conductance and susceptance are in excellent agreement with their expected hardwall value of zero. It should be noted that anomalous susceptance results are present at $400 \mathrm{and} 1800 \mathrm{~Hz}$. However, correct conductance values are obtained and it is anticipated that an improvement in susceptance could be obtained with further investigation. Since the CDL approach is intended to be used in conjunction with higher-fidelity methods, refinement to improve results at these two frequencies was not pursued. The hardwall results at $M=0.3$ presented in Figure 5 further demonstrate the applicability of the CDL approach. Here, excellent agreement is obtained 
for the normalized conductance and susceptance that extends across all frequencies.

Having obtained increased confidence from the hardwall insert cases, the eduction results for the conventional liner are considered next. In Figure 6, the educed normalized resistance and reactance for the CDL approach are compared with those from CHE and KT. With the exception of a few slight discrepancies, the CDL approach appears to perform quite well. The presence of higher order modes above $2600 \mathrm{~Hz}$ is a likely explanation for the small differences at the higher frequencies. At the low end of the frequency spectrum $(400 \mathrm{~Hz})$, the CDL and CHE resistance values are similar and show a slight increase. Conversely, the KT algorithm failed to converge. This behavior is not entirely unexpected, as the frequency is approaching an antiresonant value. As discussed by Watson, 11 this is consistent with previous findings in which low attenuation associated with an impedance near antiresonance causes diffuse objective function contours and increased uncertainty in educed resistances. There is also some slight discrepancy in the reactance at $1600-1800 \mathrm{~Hz}$ (frequencies near resonance). Overall, the agreement between the CDL method and the other methods is quite promising, in spite of the fact that the CDL method does not account for reflections from the leading and trailing edges of the liner. To provide further confirmation, CHE- and CDL-computed sound pressure level (SPL) and phase are compared at the microphone locations. Results for $M=0.0$ at $1000 \mathrm{~Hz}$, which are representative of profiles at other frequencies, are presented in Figure 7 As expected, the CHE SPL profile shows evidence of a standing wave, while the CDL profile does not. However, the overall attenuation of the liner appears to be well captured by CDL and the phase comparison is excellent. Thus, the CDL approach appears to capture sufficient detail to offer a good approximation to the educed impedance.

In an effort to replicate the effects of measurement uncertainty, random error was added to the simulated data at each microphone location independently. Specifically, the sound pressure level and phase at each microphone location were randomly perturbed by $+/-0.5 \mathrm{~dB}$ and $+/-1$ degree, respectively. As seen in Figure 8 , the randomization has minimal affect on the educed impedances. Again, the differences at $3000 \mathrm{~Hz}$ are likely the result of higher order modes being present and the added input uncertainty did not greatly affect the educed impedance values.

As with the hardwall insert, the $M=0.3$ flow condition was also considered for the conventional liner. The educed normalized resistance and reactance for this case are presented in Figure 9 Again, very good comparisons are obtained with perhaps a slight improvement over the $M=0.0$ case. The representative SPL and phase profiles at $M=0.3$ and $1000 \mathrm{~Hz}$ shown in Figure 10 may provide some indication for this behavior. A standing wave pattern is again visible in the CHE SPL profile. However, it is reduced in comparison to the $M=0.0$ case and the CDL results captures the profile more closely. The phase comparison is again excellent. Similar trends are seen with the addition of random error in Figure 11 with the data uncertainty showing minimal effect and the overall results showing a slight improvement over the $M=0.0$ case.

It should be noted that reflections from a nonanechoic termination may cause additional difficulties with the CDL approach. If so, one possible remedy may be to set the CDL source level to that at the leading edge of the liner and consider a subset of the microphones (e.g., only those over the liner). However, bearing in mind the context in which the CDL approach is proposed to be used, the results are excellent and offer justification to pursue more complex impedance eduction cases.

\section{Concluding Remarks}

Currently available impedance eduction methods have, in general, provided excellent results that continue to be used throughout the acoustic liner community. However, the applications have typically involved uniform impedance acoustic liners such that two-dimensional or quasi-three-dimensional models could be employed. To achieve the aforementioned noise reduction goals, advanced broadband liner designs will likely depart from conventional uniform impedance configurations (e.g., zone liners, variable impedance liners). Therefore, educing the impedance of these axial- and/or spanwise-variable impedance liners will require models that account for three-dimensional effects. While this capability exists with methods based on the convected Helmholtz and linearized Euler equations, an increase in computational requirements is inherently incurred.

This paper describes an extension of the use of the CDL code to acoustic liner impedance eduction or admittance, in the case of high impedance values, which utilizes a parabolic approximation to the convected Helmholtz equation. This affords very efficient propagation calculations, thus allowing solutions for complex three-dimensional geometries to be handled with relatively low computational costs. However, this efficiency can come at the expense of reduced fidelity, particularly as reflection of acoustic waves become important. Nevertheless, with the effects of the parabolic approximation in mind, CDL can provide an efficient framework in which to perform fully three-dimensional aeroacoustic computations. It has been successfully coupled with the optimization package of the SciPy ${ }^{77}$ library and used effectively in several broadband liner optimization studies. $\frac{8}{10}$ Thus, it would seem advantageous to use CDL in a 
coupled fashion with the finite element codes employed in the eduction methods based on the convected Helmholtz and linearized Euler equations. This could take the form of a multifidelity modeling framework (e.g., using surrogate models) and/or simply using CDL to efficiently identify initial values for the higher fidelity, albeit more computationally intensive, methods.

In this work, the proposed CDL impedance eduction approach is applied to a hardwall insert and conventional liner using simulated data. Educed admittances from the CDL approach compare well with expected values for the hardwall insert, as well as impedance values educed for the conventional liner using two extensively tested and validated approaches. The results are very promising and provide justification to further pursue the complementary use of CDL with the currently used finite element codes to increase the efficiency of the eduction process for configurations involving three-dimensional effects.

\section{Acknowledgments}

This research was funded by the Advanced Air Transport Technology (AATT) Project of the NASA Advanced Air Vehicles Program (AAVP).

\section{References}

${ }^{1}$ Jones, M. G. and Watson, W. R., "On the Use of Experimental Methods to Improve Confidence in Educed Impedance," AIAA Paper 20112865, 2011. 2001.

${ }^{2}$ Watson, W. R., Tracy, M. B., Jones, M. G., and Parrott, T. L., "Impedance Eduction in the Presence of Shear Flow," AIAA Paper 2001-2263,

${ }^{3}$ Jones, M. G., Watson, W. R., and Nark, D. M., "Effects of Flow Profile on Educed Acoustic Liner Impedance," AIAA Paper 2010-3763, 2010.

${ }^{4}$ Nark, D. M., Farassat, F., Pope, D. S., and Vatsa, V., "The Development of the Ducted Fan Noise Propagation and Radiation Code CDUCTLaRC," AIAA Paper 2003-3242, 2003.

${ }^{5}$ Dougherty, R. P., "A Wave-Splitting Technique for Nacelle Acoustic Propagation,” AIAA Paper 97-1652, 1997.

${ }^{6}$ Dougherty, R. P., "A Parabolic Approximation for Flow Effects on Sound Propagation in Nonuniform, Softwall, Ducts," AIAA Paper 99$1822,1999$.

${ }^{7}$ Jones, E., Oliphant, T., Peterson, P., et al., "SciPy: Open source scientific tools for Python," 2001 -.

${ }^{8}$ Nark, D. M. and Jones, M. G., "Broadband Liner Optimization for the Source Diagnostic Test Fan,” AIAA Paper 2012-2195, 2012.

${ }^{9}$ Nark, D. M., Jones, M. G., and Sutliff, D. L., "Modeling of Broadband Liners Applied to the Advanced Noise Control Fan," AIAA Paper 2015-2693, 2015.

${ }^{10}$ Nark, D. M., Jones, M. G., and Sutliff, D. L., "Further Development and Assessment of a Broadband Liner Optimization Process," AIAA Paper 2016-2784, 2016.

${ }^{11}$ Watson, W. R. and Jones, M. G., "New Numerical Procedure for Impedance Eduction in Ducts Containing Mean Flow," AIAA Journal, Vol. 49, No. 10, Oct. 2011, pp. 2109-2122.

${ }^{12}$ Watson, W. R., Carpenter, M. H., and Jones, M. G., "Performance of Kumaresan and Tufts Algorithm in Liner Impedance Eduction with Flow," AIAA Journal, Vol. 53, No. 4, April 2015, pp. 1091-1102.

${ }^{13}$ Parrott, T. L. and Jones, M. G., "Assessment of NASA's Aircraft Noise Prediction Capability, Chapter 6: Uncertainty in Acoustic Liner Impedance Measurement and Prediction," NASA TP 2102-215653, July 2012.

${ }^{14}$ Jones, M. G., Watson, W. R., Parrott, T. L., and Smith, C. D., "Design and Evaluation of Modifications to the NASA Langley Flow Impedance Tube," AIAA Paper 2004-2837, 2004.

${ }^{15}$ Jones, M. G., Watson, W. R., and June, J. C., “Optimization of Microphone Locations for Acoustic Liner Impedance Eduction,” AIAA Paper 2015-3271, 2015.

${ }^{16}$ Watson, W. R. and Jones, M. G., "Comparison of a Convected Helmholtz and Euler Model for Impedance Eduction in Flow," AIAA Paper 2006-2643, 2006.

${ }^{17}$ Watson, W. R. and Jones, M. G., "Impedance Eduction in Ducts with Higher-Order Modes and Flow," AIAA Paper 2009-3236, 2009.

${ }^{18}$ Jones, M. G. and Watson, W. R., "Explanation of Anomalous Behavior Observed in Impedance Eduction Techniques Using Measured Data," AIAA Paper 2010-3766, 2010.

${ }^{19}$ Watson, W. R. and Jones, M. G., "A Comparative Study of Four Impedance Eduction Methodologies Using Several Test Liners," AIAA Paper 2013-2274, 2013.

${ }^{20}$ Watson, W. R. and Jones, M. G., "Impedance Eduction in Sound Fields with Peripherally Varying Liners and Flow," AIAA Paper 2015-2228, 2015.

${ }^{21}$ Lan, J. H., "Turbofan Duct Propagation Model,” NASA CR 2001-211245, 2001.

${ }^{22}$ Byrd, R. H., Lu, P., and Nocedal, J., "A Limited Memory Algorithm for Bound Constrained Optimization,” SIAM Journal on Scientific and Statistical Computing, Vol. 16, No. 5, 1995, pp. 1190-1208.

${ }^{23}$ Zhu, C., Byrd, R. H., and Nocedal, J., "L-BFGS-B: Algorithm 778: L-BFGS-B, FORTRAN routines for large scale bound constrained optimization," ACM Transactions on Mathematical Software, Vol. 23, No. 4, 1997, pp. 550-560. 


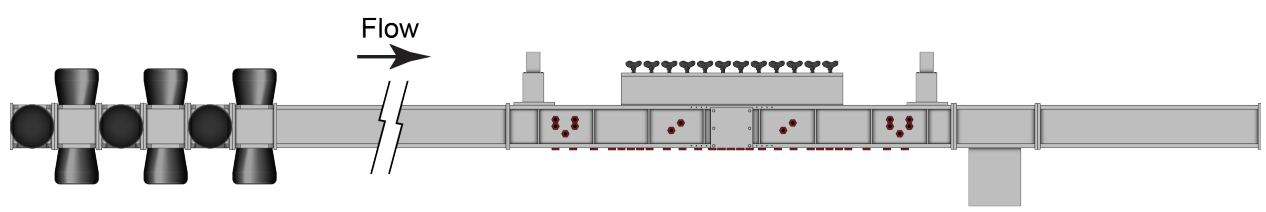

Figure 1: Sketch of Grazing Flow Impedance Tube (GFIT).

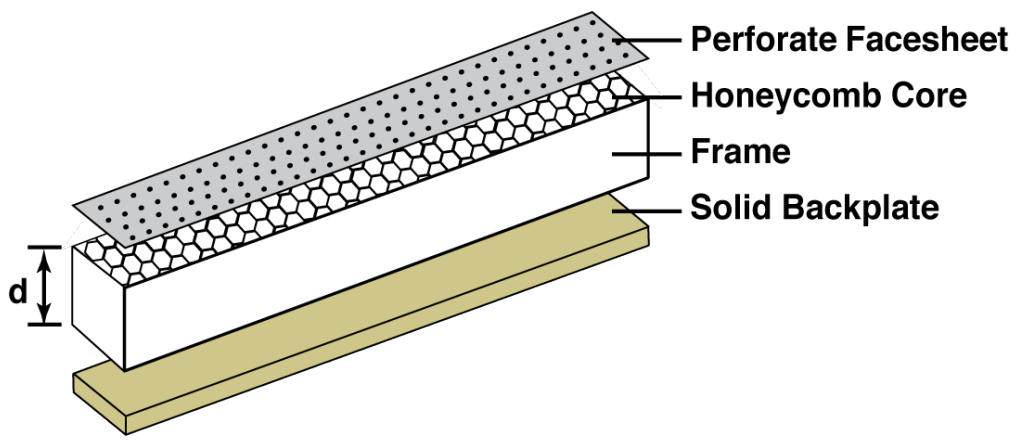

Figure 2: Sketch of GFIT liner sample.

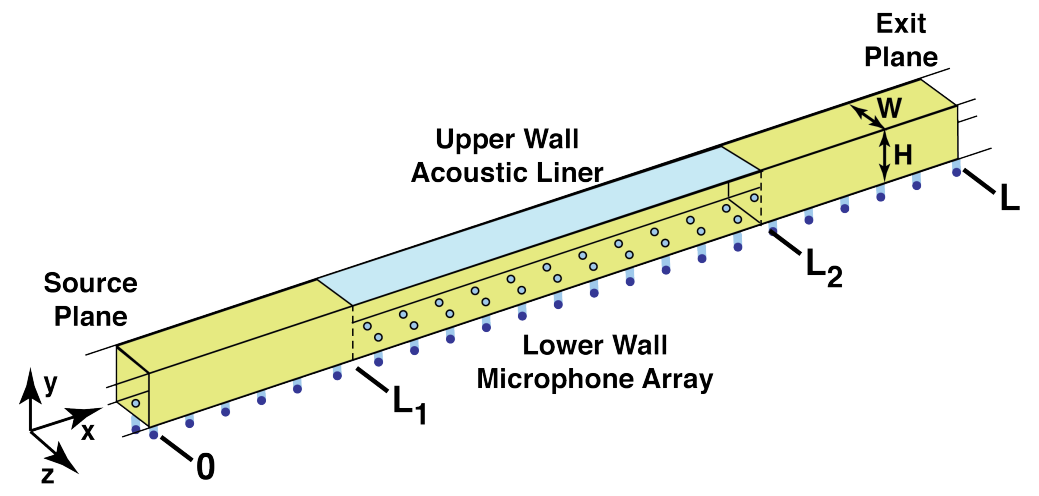

Figure 3: Sketch of GFIT computational domain. 


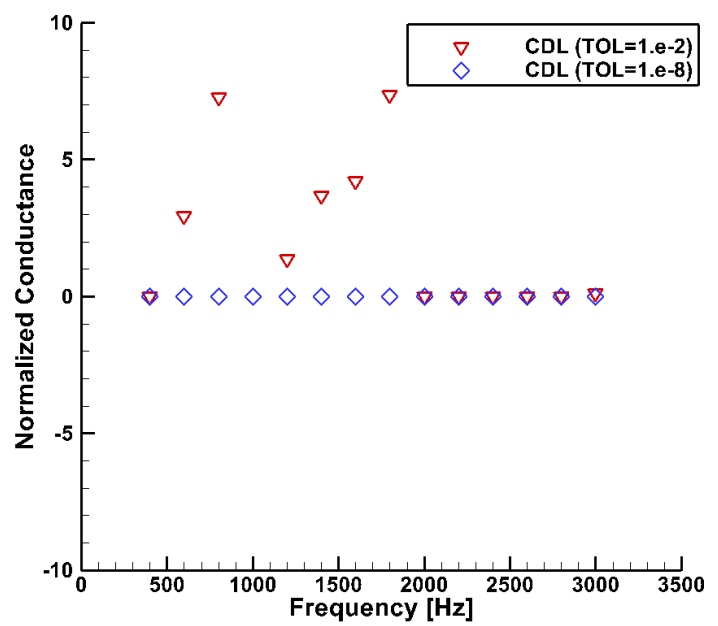

(a) Normalized Conductance

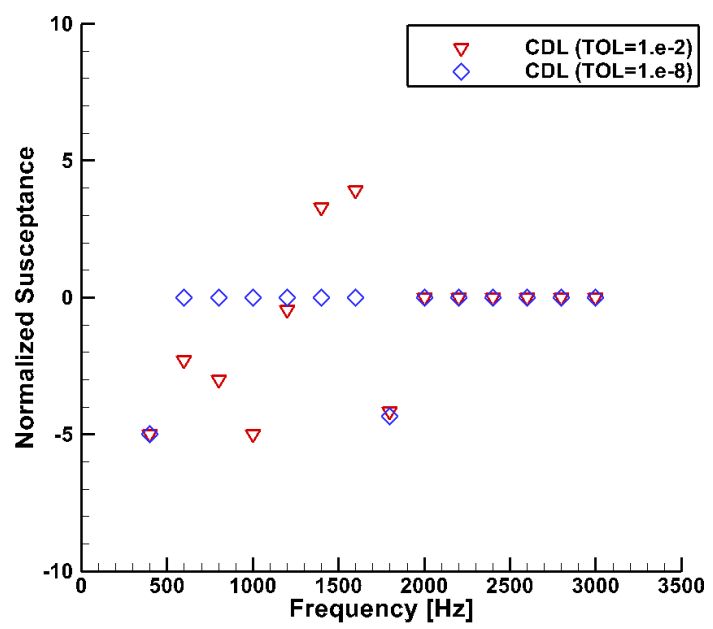

(b) Normalized Susceptance

Figure 4: Educed normalized admittance for the hardwall insert at Mach 0.0 using data generated for the GFIT.

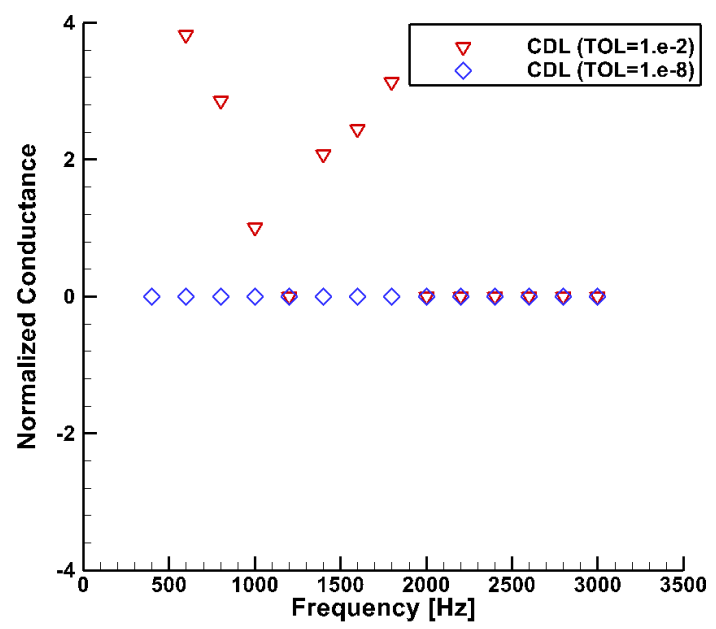

(a) Normalized Conductance

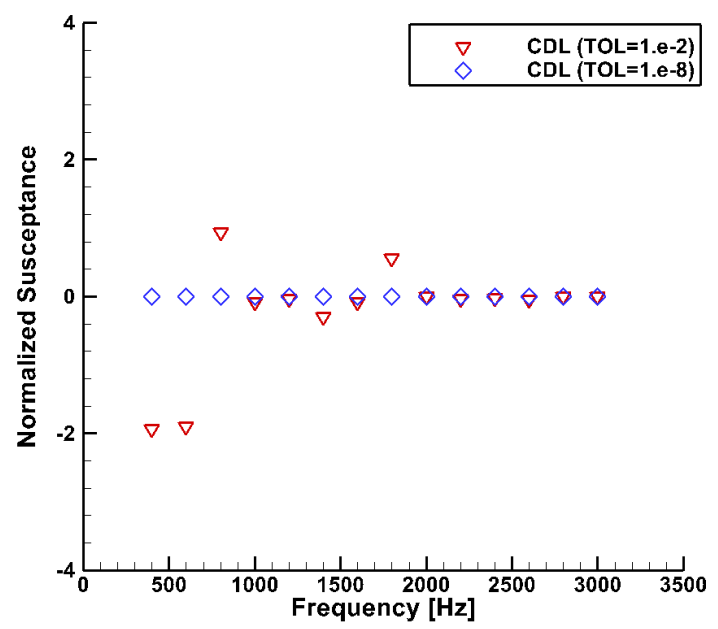

(b) Normalized Susceptance

Figure 5: Educed normalized admittance for the hardwall insert at Mach 0.3 using data generated for the GFIT. 


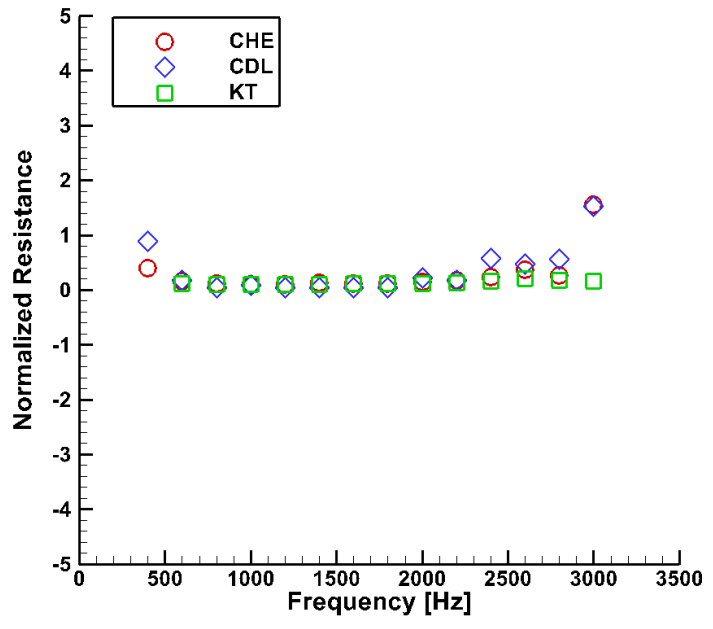

(a) Normalized Resistance

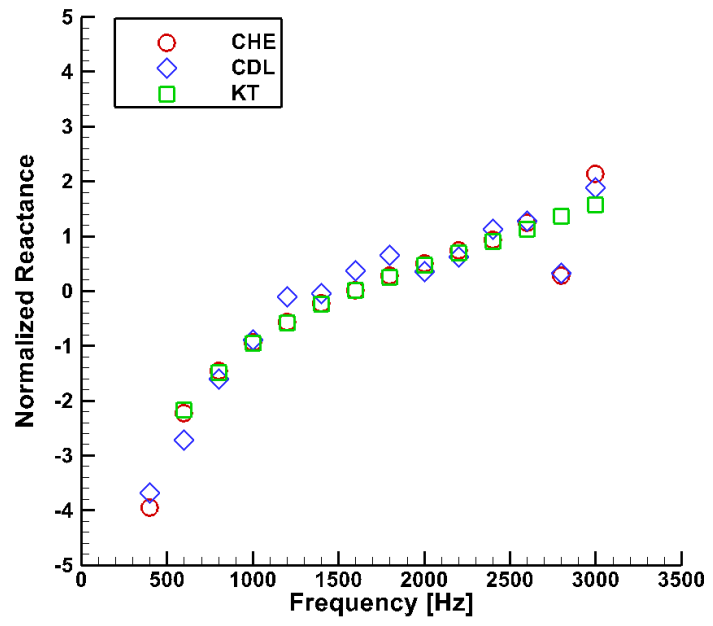

(b) Normalized Reactance

Figure 6: Educed normalized impedance for the SDOF liner at Mach 0.0 using data generated for the GFIT.

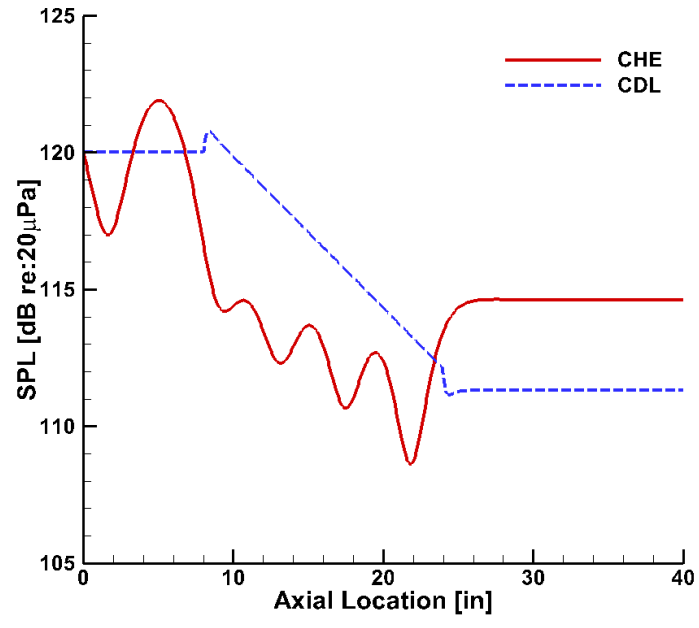

(a) Sounds Pressure Level

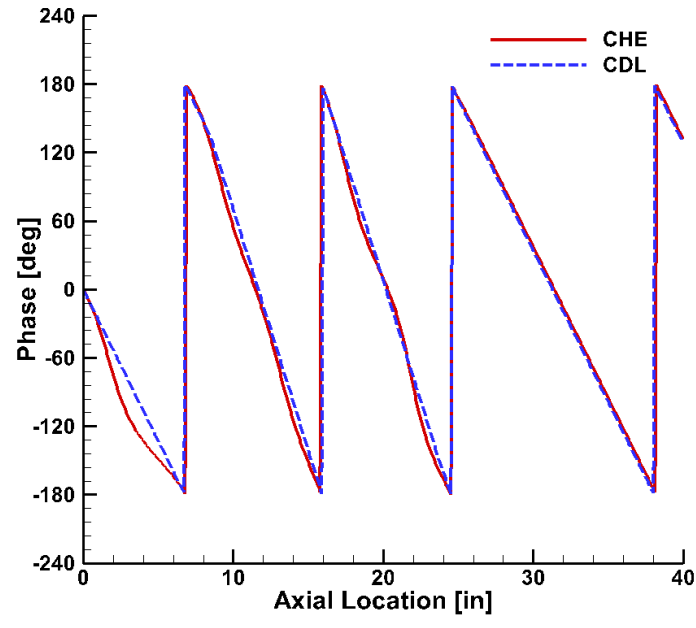

(b) Phase

Figure 7: Sound pressure level and phase axial profiles for the SDOF liner with a $1000 \mathrm{~Hz}$ plane wave source at Mach 0.0 in the GFIT. 


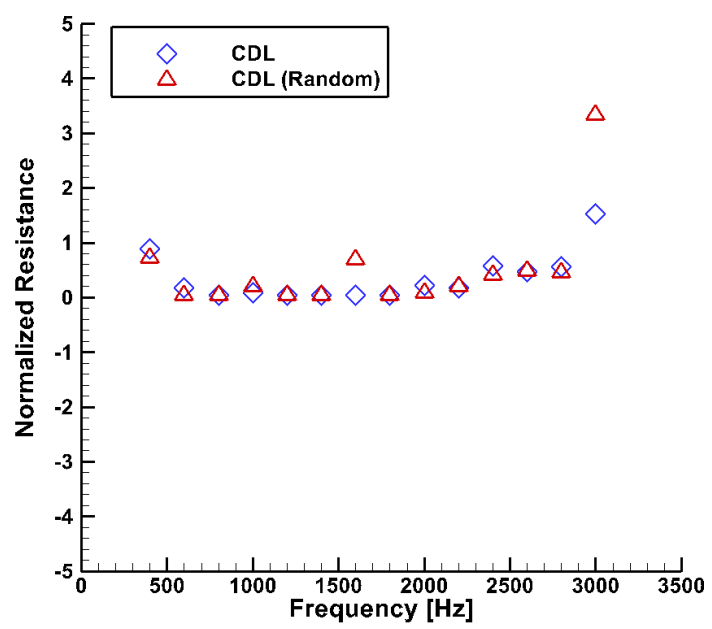

(a) Normalized Resistance

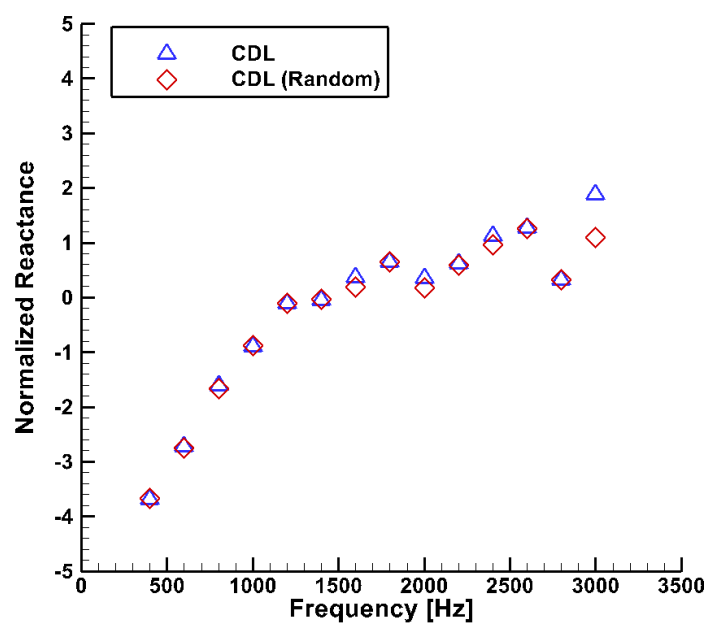

(b) Normalized Reactance

Figure 8: Educed normalized impedance for the SDOF liner at Mach 0.0 using data generated for the GFIT.

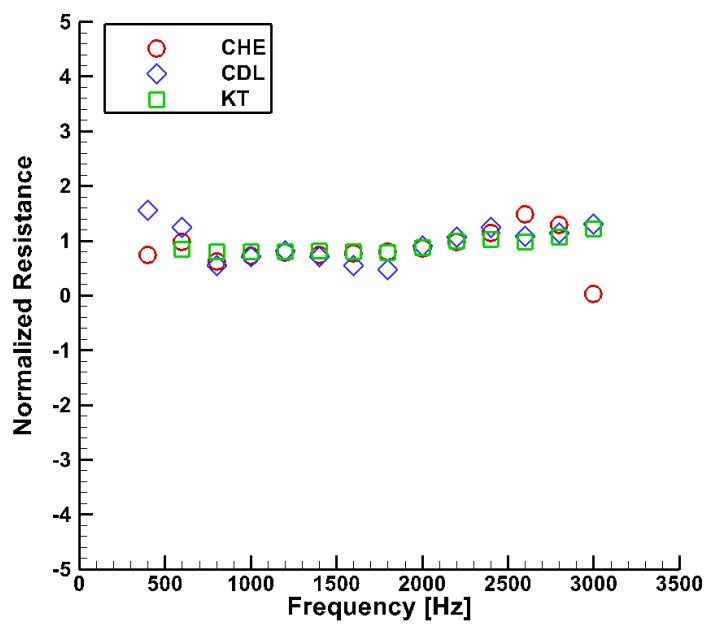

(a) Normalized Resistance

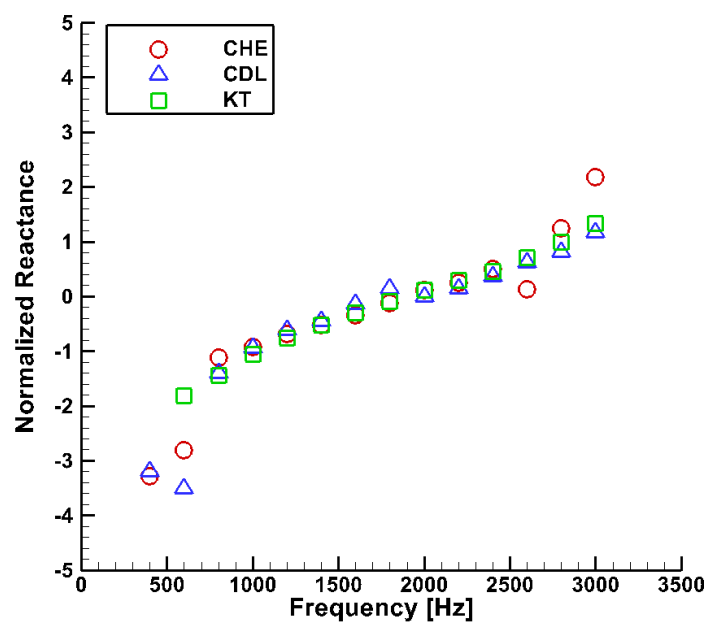

(b) Normalized Reactance

Figure 9: Educed normalized impedance for the SDOF liner at Mach 0.3 using data generated for the GFIT. 


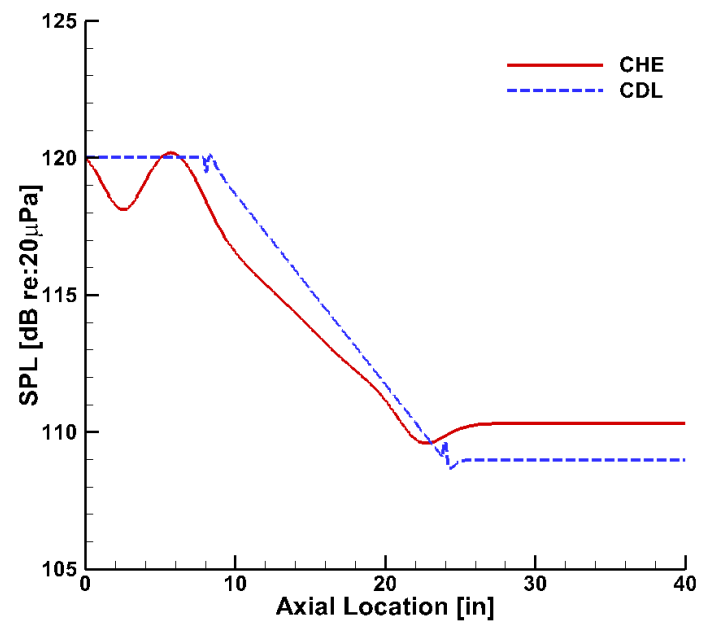

(a) Sounds Pressure Level

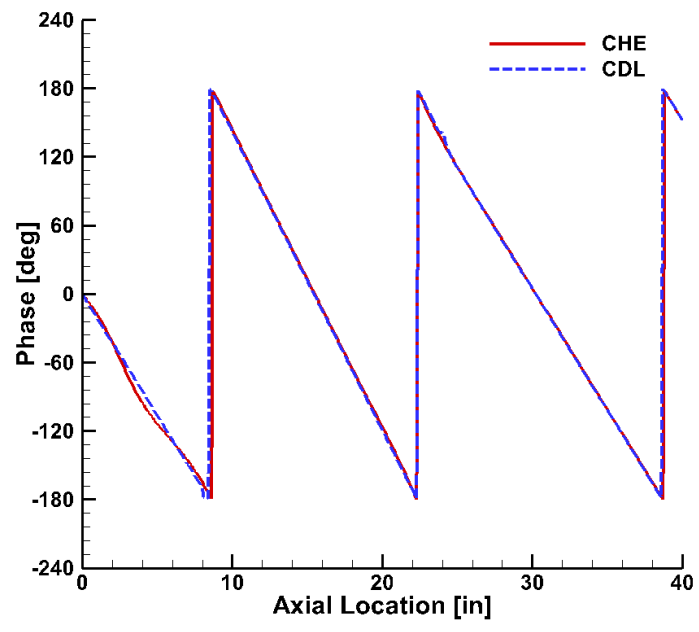

(b) Phase

Figure 10: Sound pressure level and phase axial profiles for the SDOF liner with a $1000 \mathrm{~Hz}$ plane wave source at Mach 0.3 in the GFIT.

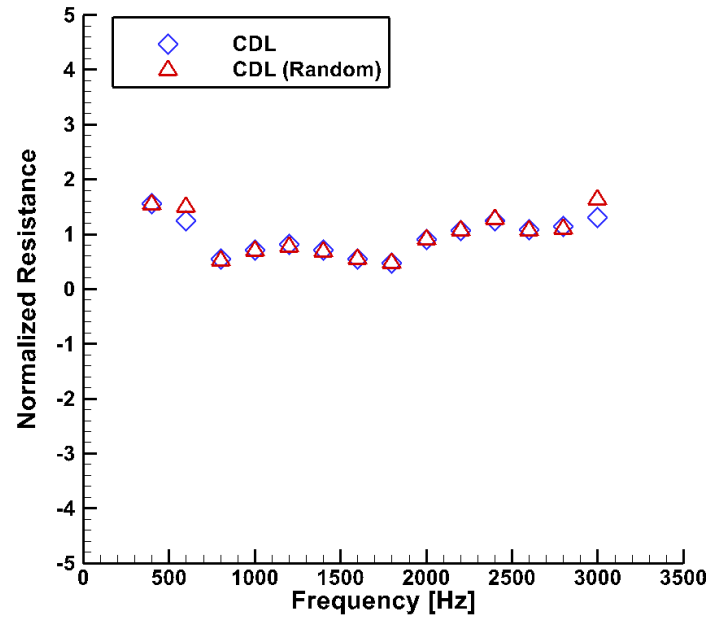

(a) Normalized Resistance

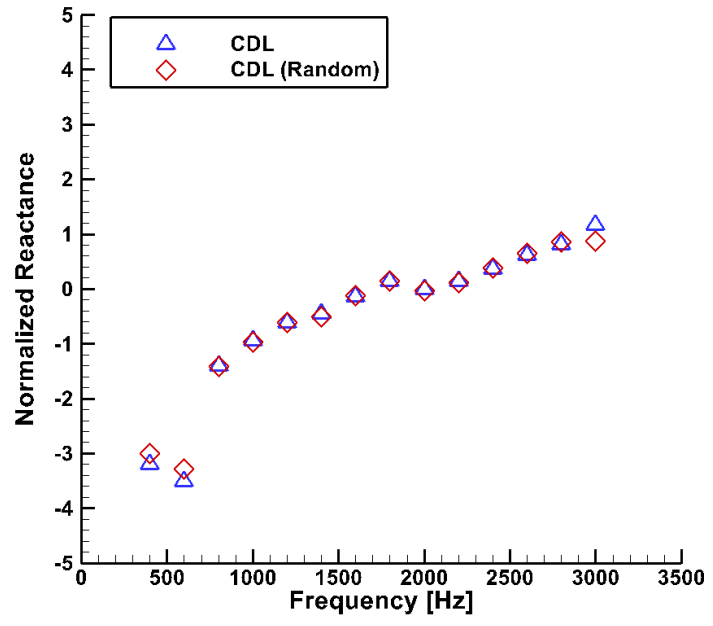

(b) Normalized Reactance

Figure 11: Educed normalized impedance for the SDOF liner at Mach 0.3 using data generated for the GFIT. 